\title{
Decentralized Networked Control System Design Using Takagi-Sugeno (TS) Fuzzy Approach
}

\author{
Chedia Latrach $^{1} \quad$ Mourad Kchaou ${ }^{2} \quad$ Abdelhamid Rabhi ${ }^{1} \quad$ Ahmed El Hajjaji $^{1}$ \\ ${ }^{1}$ Laboratory of Modelisation, Information and System, University of Picardie Jules Verne, 7 New Mill Street, Amiens, France \\ ${ }^{2}$ Electrical Engineering Department, National Engineering School of Sfax, University of Sfax, Tunisia
}

\begin{abstract}
This paper proposes a new method for control of continuous large-scale systems where the measures and control functions are distributed on calculating members which can be shared with other applications and connected to digital network communications. At first, the nonlinear large-scale system is described by a Takagi-Sugeno (TS) fuzzy model. After that, by using a fuzzy LyapunovKrasovskii functional, sufficient conditions of asymptotic stability of the behavior of the decentralized networked control system (DNCS), are developed in terms of linear matrix inequalities (LMIs). Finally, to illustrate the proposed approach, a numerical example and simulation results are presented.
\end{abstract}

Keywords: Continuous large-scale systems, decentralized static output feedback fuzzy control, networked control systems (NCS), Takagi-Sugeno (TS) fuzzy model, linear matrix inequalities (LMIs).

\section{Introduction}

Decentralized control of large-scale systems (also known as interconnected systems in some books) has been investigated as a branch of control theory and has received considerable attention over the past three decades due to its various applications such as power systems, aerospace systems, nuclear reactors, systems control process, etc. ${ }^{[1-3]}$

In fact, various techniques for distributed control using linear matrix inequalities (LMIs) were recently studied ${ }^{[4-9]}$. The systems consist of a large set of interconnected subsystems which can be far from each other. That's why we introduce the notion of communication network to connect them, and thus it aims to ensure data transmission and coordinating manipulation among spatially distributed components. Compared with conventional point-to-point control systems, the advantages of networked control systems (NCS) are less wiring, lower installation cost as well as greater agility in diagnosis and maintenance. Because of these distinctive benefits, typical application of these systems ranges over various fields, such as automotive, mobile robotics, advanced aircraft, etc. It is well known that limited network resources, network-induced delays and data packets dropout through the network, may degrade the decentralized networked control system (DNCS) performance and lead to instability. It is mentioned that the communication delay, which has time-varying characteristics, is one of the important factors to be considered in NCS analysis and synthesis ${ }^{[10-19]}$.

In this paper, the decentralized static output feedback

Regular Paper

Special Issue on Advances in Nonlinear Dynamics and Control

Manuscript received March 25, 2014; accepted September 25, 2014

Recommended by Associate Editor Fernando Tadeo

(C) Institute of Automation, Chinese Academy of Science and Springer-Verlag Berlin Heidelberg 2015 control method for stabilization of nonlinear interconnected system, that takes into account problems of delay and data packets dropout in communication, is proposed. Based on Takagi-Sugeno (TS) fuzzy system, the static output feedback controller is designed. The sufficient condition is offered to guarantee the stability of the closed-loop system using Lyapunov Krasovskii functional. Its constructive conditions are presented in LMIs terms, taking effects of communication network into account.

The paper is organized as follows. Section 2 presents system description and preliminaries. Section 3 presents the main results, describing the control strategy for largescale systems through a communication network. Section 4 shows simulation results. Finally, conclusions are given in Section 5 .

Notations. $\operatorname{sym}(W)$ stands for $W+W^{\mathrm{T}}$. The symbol $(*)$ within a matrix represents the symmetric entries.

\section{Preliminaries and system description}

Consider a large-scale system $S$ composed of $J$ interconnected subsystems $S_{i}, i=1,2, \cdots, J$. The $i$-th fuzzy subsystem $S_{i}$ is described by the following TS fuzzy model:

$$
S_{i}:\left\{\begin{array}{l}
\text { If } \theta_{i 1}(t) \text { is } F_{i 1}^{l} \text { and } \theta_{i g}(t) \text { is } F_{i g}^{l} \\
\text { then } \dot{x}_{i}(t)=A_{i}^{l} x_{i}(t)+B_{i}^{l} u_{i}(t)+\sum_{j=1}^{J} f_{i j}\left(x_{j}(t)\right) \\
y_{i}(t)=C_{2 i} x_{i}(t)
\end{array}\right.
$$

where $i=1,2, \cdots, J, l=1,2, \cdots, r_{i}, x_{i}(t)$ denotes the state vector, $y_{i}(t)$ denotes the measured output, $u_{i}(t)$ is the control input, $A_{i}^{l}, B_{i}^{l}$ and $C_{2 i}$ are constant real matrices with appropriate dimensions and $C_{2 i}$ is full rank, $\theta_{i 1}(t)$, 
$\theta_{i 2}(t), \cdots, \theta_{i g}(t)$ are some measurable premise variables for subsystems $S_{i}, F_{i q}^{l}(q=1,2, \cdots, g)$ represents the linguistic fuzzy sets of the rule, $f_{i j}\left(x_{j}(t)\right)$ represents the interconnection of fuzzy rules in subsystem $S_{i}$ and subsystem $S_{j}$, and $r_{i}$ represents the number of fuzzy rules in subsystem $S_{i}$.

Using the central-average defuzzifier, the TS fuzzy system can be given as

$$
\left\{\begin{array}{l}
\dot{x}_{i}(t)=\sum_{l=1}^{r_{i}} h_{i}^{l}\left(\theta_{i}(t)\right)\left[A_{i}^{l} x_{i}(t)+B_{i}^{l} u_{i}(t)+\sum_{j=1}^{J} f_{i j}\left(x_{j}(t)\right)\right] \\
y_{i}(t)=C_{2 i} x_{i}(t)
\end{array}\right.
$$

where

$$
\begin{array}{r}
h_{i}^{l}\left(\theta_{i}(t)\right)=\frac{v_{i}^{l}\left(\theta_{i}(t)\right)}{\sum_{l=1}^{r_{i}} v_{i}^{l}\left(\theta_{i}(t)\right)} \\
v_{i}^{l}\left(\theta_{i}(t)\right)=\prod_{q=1}^{g} F_{i q}^{l}\left(\theta_{i q}(t)\right)
\end{array}
$$

with $F_{i q}^{l}\left(\theta_{i q}(t)\right)$ as the grade of membership of $\theta_{i q}(t)$ in the fuzzy set $F_{i q}^{l} . h_{i}^{l}\left(\theta_{i}(t)\right)$ is the membership function for each fuzzy rule, which represents normalized grade of membership, and satisfies

$$
0 \leq h_{i}^{l}\left(\theta_{i}(t)\right) \leq 1, \text { for } l=1,2, \cdots, r_{i}, \sum_{l=1}^{r_{i}} h_{i}^{l}\left(\theta_{i}(t)\right)=1 .
$$

We assume that system $S$ will be controlled through network. Fig. 1 represents the structure of networked control sub system $S_{i}$ with induced delays, where $\tau_{s c i}$ is sensor-tocontroller delay and $\tau_{c a i}$ is the controller-to-actuator delay. It is assumed that the controller computational delay can be absorbed into either $\tau_{s c i}$ or $\tau_{c a i}$.

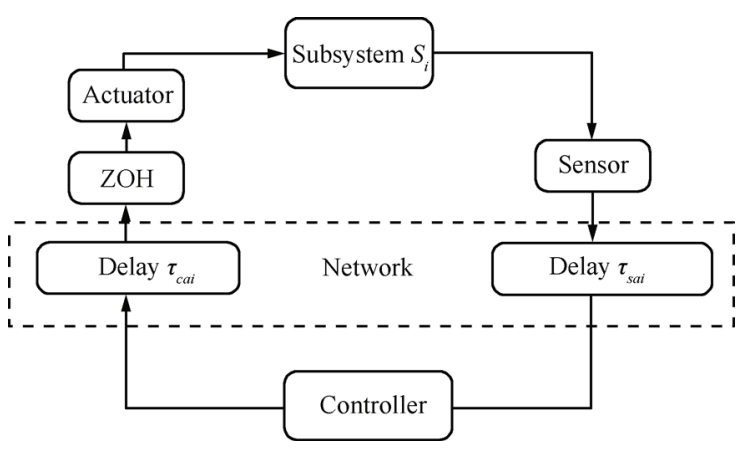

Fig. 1 Framework of networked control subsystem $S_{i}$

Assumption 1. All pairs $\left(A_{i}^{l}, B_{i}^{l}\right) \quad(i=1,2, \cdots, J$ and $\left.l=1,2, \cdots, r_{i}\right)$ are stabilizable. ${ }^{[20]}$

Assumption 2. The interconnection $f_{i j}\left(x_{j}(t)\right)$ satisfies the following conditions: $f_{i j}\left(x_{j}(t)\right)=B_{i}^{l} f_{i j^{l}}\left(x_{j}(t)\right)$ and $\left\|f_{i j}^{l}\left(x_{j}(t)\right)\right\| \leq \bar{f}_{i j}^{l}\left\|x_{j}(t)\right\|$, where $\bar{f}_{i i}^{l}=0, \bar{f}_{i j}^{l}(i \neq j)$ is a positive constant and $B_{i}^{l}$ is a constant real matrix with appropriate dimensions. ${ }^{[20]}$

Assumption 3. The sensors are clock driven, the controller and actuators are event driven.
Assumption 4. Data, either from measurement or for control, are transmitted in a single packet.

Assumption 5. The effect of signal quantization is not considered.

Assumption 6. The real input $u_{i}(t)$ for each subsystem, realized through a zero-order hold $(\mathrm{ZOH})$, is a piecewise constant function.

It is worth mentioning that the sampling period of a sensor is pre-determined for control algorithm design, and thus the sensor can be assumed to be clock driven. However, an actuator does not change its output to the plant under control until an updated control signal is received, implying that the actuator is event driven.

To obtain our main results, the following lemmas are needed.

Lemma 1. ${ }^{[21]}$ For each real vector $\zeta$ and $\rho$, it follows that

$$
2 \zeta^{\mathrm{T}} \rho \leq \zeta^{\mathrm{T}} Z \zeta+\rho^{\mathrm{T}} Z^{-1} \rho
$$

with $Z>0$.

Lemma 2. ${ }^{[20]}$ The following inequality is verified for each real vector $\nu_{i} \in \mathbf{R}^{n}$ :

$$
\left[\sum_{i=1}^{m} \nu_{i}\right]\left[\sum_{i=1}^{\mathrm{T}} \nu_{i}\right] \leq m \sum_{i=1}^{m} \nu_{i}^{\mathrm{T}} \nu_{i}
$$

\section{Main results}

In this section, we are interested in the design of static output feedback controller in order to stabilize the system. Indeed, it is assumed that the states of the system (2) are not all available for measurement, that is why we achieve an output feedback control. The control scheme type parallel distributed compensation (PDC) will be considered for each subsystem $S_{i}$. The overall fuzzy PDC networked controller corresponding to $S_{i}$ can be described as

$$
u_{i}\left(t_{k}\right)=\sum_{l=1}^{r_{i}} h_{i}^{l}\left(\theta_{i}\left(t_{k}\right)\right) K_{i}^{l} y_{i}\left(t_{k}-\tau_{k i}\right) .
$$

From the $\mathrm{ZOH}$, the input signal for each subsystem $S_{i}$ for $t_{k} \leq t \leq t_{k+1}$ is given by

$$
u_{i}(t)=\sum_{l=1}^{r_{i}} h_{i}^{l}\left(\theta_{i}\left(t_{k}\right)\right) K_{i}^{l} y_{i}\left(t_{k}-\tau_{k i}\right) .
$$

For network-induced delay $\left(\tau_{k i}\right)$, one major challenge for NCS design is the effect of network-induced delays in a control loop. It occurs when the system components exchange data across the network. It can degrade control performance significantly or even destabilize the system. The delays in NCS consist of a communication delay between sensors and controllers $\tau_{\text {sci }}$, a communication delay between controller and actuators $\tau_{c a i}$, computational time in controller $\tau_{c}$ which can be generally included in the controller to actuator delay.

A natural assumption on $\tau_{k i}$ can be made as

$$
0<\tau_{m i} \leq \tau_{k i} \leq \tau_{M i} .
$$


Packet dropouts are network-induced effects which can be the consequence of a link failure. They can also be generated purposefully in order to avoid congestion or to guarantee the most recent data to be sent. Although most network protocols are equipped with transmission-retry mechanisms, they can only re-transmit for limited time. After this time has expired, the packets are dropped. Normally, feedback controllers can tolerate a certain amount of packet losses. But the consecutive packet losses have an adverse impact on the overall performance.

$$
t_{k+1}-t_{k}=\bar{\sigma}_{i} T_{e}+\max _{i}\left\{\tau_{(k+1) i}\right\}-\min _{i}\left\{\tau_{k i}\right\}
$$

where $T_{e}$ denotes the sampling period, $t_{k}$ denotes the sampling instant, and $\bar{\sigma}_{i}$ denotes the maximum number of packet dropouts in the updating periods. Using (2) and (7), the closed-loop networked control system can be written for $t_{k} \leq t \leq t_{k+1}$ as

$$
\left\{\begin{array}{l}
\dot{x}_{i}(t)=A(t) x_{i}(t)+H(t) x_{i}\left(t_{k}-\tau_{k i}\right)+f\left(x_{i}(t)\right) \\
y_{i}(t)=C_{2 i} x_{i}(t)
\end{array}\right.
$$

with

$$
\begin{aligned}
A(t) & =\sum_{l=1}^{r_{i}} h_{i}^{l} A_{i}^{l} \\
B(t) & =\sum_{l=1}^{r_{i}} h_{i}^{l} B_{i}^{l} \\
H(t) & =B(t) \sum_{s=1}^{r_{i}} h_{i}^{s} K_{i}^{s} C_{2 i} \\
f\left(x_{i}(t)\right) & =\sum_{l=1}^{r_{i}} h_{i}^{l} \sum_{j=1}^{J} f_{i j}\left(x_{j}\right) .
\end{aligned}
$$

Defining $\eta_{i}(t)=t-t_{k}+\tau_{k i}, \quad t_{k} \leq t \leq t_{k+1}$, then

$$
\tau_{k i} \leq \eta_{i}(t) \leq \bar{\sigma}_{i} T_{e}+\max _{i}\left\{\tau_{(k+1) i}\right\}
$$

Thus, we get from [22] that

$$
\eta_{1 i} \leq \eta_{i}(t) \leq \eta_{2 i}, \quad \dot{\eta}_{i}(t) \leq h_{d i}
$$

where

$$
\eta_{1 i}=\tau_{m i} \quad \text { and } \quad \eta_{2 i}=\bar{\sigma}_{i} T_{e}+\max _{i}\left\{\tau_{M i}\right\} .
$$

As $\sum_{k=0}^{\infty}\left[t_{k}, t_{k+1}\right)=[0, \infty)$, we have

$$
\left\{\begin{array}{l}
\dot{x}_{i}(t)=A(t) x_{i}(t)+H(t) x_{i}\left(t-\eta_{i}(t)\right)+f\left(x_{i}(t)\right) \\
y_{i}(t)=C_{2 i} x_{i}(t) \\
x_{i}(t)=\phi_{i}(t), t \in\left[t_{0}-\eta_{2 i}, t_{0}\right]
\end{array}\right.
$$

where $\phi_{i}(t)$ can be viewed as the initial condition of the closed-loop control system. Then based on (12), it is noted that the NCS (13) is equivalent to a system with an interval time-varying delay.

The controller design is based on the following preliminary result given by the Lemma 3 .

Lemma 3. For given scalars $\eta_{1 i}>0$ and $\eta_{2 i}>0$, the closed-loop system (13) is asymptotically stable, if there exist positive matrices $P_{i}, Q_{1 i}, Q_{2 i}, Q_{3 i}, Z_{1 i}$, and matrices $G_{1 i}, G_{2 i}$ and $G_{3 i}$, with appropriate dimensions, such that the following conditions hold:

$$
\Phi_{i j}=\left[\begin{array}{ccccc}
\Phi_{11 i j} & \Phi_{12 i} & Z_{1 i} & 0 & \Phi_{15 i} \\
* & \Phi_{22 i} & 0 & 0 & \Phi_{25 i j} \\
* & * & -Q_{2 i}-Z_{1 i} & 0 & 0 \\
* & * & * & -Q_{3 i} & 0 \\
* & * & * & * & \Phi_{55 i}
\end{array}\right]<0
$$

$$
\begin{aligned}
\Phi_{11 i j}= & Q_{1 i}+Q_{2 i}+Q_{3 i}+\operatorname{sym}\left(G_{1 i}^{\mathrm{T}} A(t)\right)-Z_{1 i}+G_{1 i}^{\mathrm{T}} G_{1 i}+ \\
& \left(3 J \sum_{j=1}^{J} \hat{f}_{j i}^{2}\left\|\hat{B}_{j}\right\|^{2}\right) I \\
\Phi_{12 i}= & A(t)^{\mathrm{T}} G_{2 i}+G_{1 i}^{\mathrm{T}} H(t) \\
\Phi_{22 i}= & \operatorname{sym}\left(G_{2 i}^{\mathrm{T}} H(t)\right)-\left(1-h_{d i}\right) Q_{1 i}+G_{2 i}^{\mathrm{T}} G_{2 i} \\
\Phi_{15 i}= & P_{i}-G_{1 i}^{\mathrm{T}}+A(t)^{\mathrm{T}} G_{3 i} \\
\Phi_{25 i j}= & -G_{2 i}^{\mathrm{T}}+H^{\mathrm{T}}(t) G_{3 i} \\
\Phi_{55 i}= & \eta_{1 i}^{2} Z_{1 i}-\operatorname{sym}\left(G_{3 i}\right)+G_{3 i}^{\mathrm{T}} G_{3 i} .
\end{aligned}
$$

Proof. Let the Lyapunov-Krasovskii functional candidate be

$$
V(t)=\sum_{i=1}^{J} v_{i}(t), i=1,2, \cdots, J
$$

where $v_{i}(t)$ denotes the Lyapunov-Krasovskii functional corresponding to fuzzy subsystem $S_{i}$. Each $v_{i}(t)$ is defined as

$$
\begin{aligned}
v_{i}(t) & =x_{i}^{\mathrm{T}}(t) P_{i} x_{i}(t)+\int_{t-\eta_{i}(t)}^{t} x_{i}^{\mathrm{T}}(s) Q_{1 i} x_{i}(s) \mathrm{d} s+ \\
& \int_{t-\eta_{1 i}}^{t} x_{i}^{\mathrm{T}}(s) Q_{2 i} x_{i}(s) \mathrm{d} s+ \\
& \int_{t-\eta_{2 i}}^{t} x_{i}^{\mathrm{T}}(s) Q_{3 i} x_{i}(s) \mathrm{d} s+ \\
& \eta_{1 i} \int_{-\eta_{1 i}}^{0}\left(\int_{t+s}^{t} \dot{x}_{i}^{\mathrm{T}}(v) Z_{1 i} \dot{x}_{i}(v) \mathrm{d} v\right) \mathrm{d} s .
\end{aligned}
$$

The corresponding time derivative of $v_{i}(t)$ is given by

$$
\begin{aligned}
\dot{v}_{i}(t) \leq & 2 \dot{x}_{i}^{\mathrm{T}}(t) P_{i} x_{i}(t)+x_{i}^{\mathrm{T}}(t)\left(Q_{1 i}+Q_{2 i}+Q_{3 i}\right) x_{i}(t)- \\
& \left(1-h_{d i}\right) x_{i}^{\mathrm{T}}\left(t-\eta_{i}(t)\right) Q_{1 i} x_{i}\left(t-\eta_{i}(t)\right)- \\
& x_{i}^{\mathrm{T}}\left(t-\eta_{1 i}\right) Q_{2 i} x_{i}\left(t-\eta_{1 i}\right)- \\
& x_{i}^{\mathrm{T}}\left(t-\eta_{2 i}\right) Q_{3 i} x_{i}\left(t-\eta_{2 i}\right)+ \\
& \dot{x}_{i}^{\mathrm{T}}(t)\left(\eta_{1 i}^{2} Z_{1 i}\right) \dot{x}_{i}(t)- \\
& \eta_{1 i} \int_{t-\eta_{1 i}}^{t} \dot{x}_{i}^{\mathrm{T}}(v) Z_{1 i} \dot{x}_{i}(v) \mathrm{d} v .
\end{aligned}
$$

Denoting $\psi_{1 i}=x_{i}(t)-x_{i}\left(t-\eta_{1 i}\right)$, by Jensen inequality, we can obtain

$$
-\eta_{1 i} \int_{t-\eta_{1 i}}^{t} \dot{x}_{i}^{\mathrm{T}}(v) Z_{1 i} \dot{x}_{i}(v) \mathrm{d} v \leq-\psi_{1 i}^{\mathrm{T}} Z_{1 i} \psi_{1 i} .
$$


From (13), we construct for appropriately dimensioned matrices $G_{1 i}, G_{2 i}$, and $G_{3 i}$ as the following zero-value expression:

$$
\begin{aligned}
& 2\left[x_{i}^{\mathrm{T}}(t) G_{1 i}^{\mathrm{T}}+x_{i}^{\mathrm{T}}\left(t-\eta_{i}(t)\right) G_{2 i}^{\mathrm{T}}+\dot{x}_{i}^{\mathrm{T}}(t) G_{3 i}^{\mathrm{T}}\right] \times \\
& {\left[-\dot{x}_{i}(t)+A(t) x_{i}(t)+H(t) x\left(t-\eta_{i}(t)\right)+f\left(x_{i}(t)\right)\right]=0} \\
& \Psi_{i}^{\mathrm{T}}(t)= \\
& {\left[\begin{array}{lllll}
x_{i}^{\mathrm{T}}(t) & x_{i}^{\mathrm{T}}\left(t-\eta_{i}(t)\right) & x_{i}^{\mathrm{T}}\left(t-\eta_{1 i}\right) & x_{i}^{\mathrm{T}}\left(t-\eta_{2 i}\right) & \dot{x}_{i}^{\mathrm{T}}(t)
\end{array}\right] .}
\end{aligned}
$$

According to Lemmas 1 and 2, we have

$$
\begin{aligned}
& 2 x_{i}^{\mathrm{T}}(t) G_{1 i}^{\mathrm{T}} \sum_{j=1}^{J} f_{i j}\left(x_{j}\right) \leq \\
& x_{i}^{\mathrm{T}}(t) G_{1 i}^{\mathrm{T}} G_{1 i} x_{i}(t)+\sum_{j=1}^{J} f_{i j}^{\mathrm{T}}\left(x_{j}\right) \sum_{j=1}^{J} f_{i j}\left(x_{j}\right) \leq \\
& x_{i}^{\mathrm{T}}(t) G_{1 i}^{\mathrm{T}} G_{1 i} x_{i}(t)+J \sum_{j=1}^{J} f_{i j}^{\mathrm{T}}\left(x_{j}\right) f_{i j}\left(x_{j}\right) .
\end{aligned}
$$

Based on Assumptions 1 and 2, and defining $\hat{f}_{i j}=\max _{l} \bar{f}_{i j}^{l}$, $\left\|\hat{B}_{i}\right\|=\max _{l}\left\|B_{i}^{l}\right\|$, we have

$$
\begin{aligned}
& 2 x_{i}^{\mathrm{T}}(t) G_{1 i}^{\mathrm{T}} \sum_{j=1}^{J} f_{i j}\left(x_{j}\right) \leq \\
& x_{i}^{\mathrm{T}}(t) G_{1 i}^{\mathrm{T}} G_{1 i} x_{i}(t)+J \sum_{j=1}^{J} f_{i j}^{\mathrm{T}}\left(x_{j}\right) f_{i j}\left(x_{j}\right) \leq \\
& x_{i}^{\mathrm{T}}(t)\left(G_{1 i}^{\mathrm{T}} G_{1 i}+J \sum_{j=1}^{J} \hat{f}_{j i}^{2}\left\|\hat{B}_{j}\right\|^{2}\right) I x_{i}(t) \\
& 2 x_{i}^{\mathrm{T}}\left(t-\eta_{i}(t)\right) G_{2 i}^{\mathrm{T}} \sum_{j=1}^{J} f_{i j}\left(x_{j}\right) \leq \\
& x_{i}^{\mathrm{T}}\left(t-\eta_{i}(t)\right) G_{2 i}^{\mathrm{T}} G_{2 i} x_{i}\left(t-\eta_{i}(t)\right)+ \\
& x_{i}^{\mathrm{T}}(t) J \sum_{j=1}^{J} \hat{f}_{j i}^{2}\left\|\hat{B}_{j}\right\|^{2} I x_{i}(t) \\
& 2 \dot{x}_{i}^{\mathrm{T}}(t) G_{3 i}^{\mathrm{T}} \sum_{j=1}^{J} f_{i j}\left(x_{j}\right) \leq \\
& \left.\left.\dot{x}_{i}^{\mathrm{T}}(t)\right) G_{3 i}^{\mathrm{T}} G_{3 i} \dot{x}_{i}(t)+x_{i}^{\mathrm{T}}(t) J \sum_{j=1}^{J} \hat{f}_{j i}^{2}\left\|\hat{B}_{j}\right\|^{2}\right) I x_{i}(t) .
\end{aligned}
$$

Considering (17) - (19) and (21) - (23), the derivative of (15) along the closed loop system (13) can be described as

$$
\dot{V}(t)=\sum_{i=1}^{J} \dot{v}_{i}(t) \leq \sum_{i=1}^{J} \sum_{j=1}^{J} \sum_{l=1}^{r_{i}} \sum_{s=1}^{r_{i}} h_{i}^{l} h_{j}^{s} \Psi_{i}^{\mathrm{T}}(t) \Phi_{i j} \Psi_{i}(t) \leq 0 .
$$

According to Lemma 3, we have $\dot{V}(t)<0$. So system (13) is asymptotically stable.

The objective now is to determine the gain matrices $K_{i}^{l}$ such that the static output feedback closed-loop system is asymptotically stable.
Theorem 1. For given scalars $\eta_{1 i}>0, \eta_{2 i}>0, \mu_{1}$, $\mu_{2}$, and $\mu_{3}$, the closed-loop system (13) is asymptotically stable, if there exist positive matrices $\bar{P}_{i}, \bar{Q}_{1 i}, \bar{Q}_{2 i}, \bar{Q}_{3 i}$, $\bar{Z}_{1 i}$, matrices $\hat{G}_{11 i}>0, \hat{G}_{21 i}>0, \hat{G}_{22 i}>0$, and $Y_{i}^{s}$, with appropriate dimensions, such that the following conditions hold

$$
\begin{aligned}
& \bar{\Phi}_{i j}^{l l}<0 \\
& \bar{\Phi}_{i j}^{l s}+\bar{\Phi}_{i j}^{s l}<0, \quad j>i, \quad s>l
\end{aligned}
$$

where

$$
\begin{aligned}
& \bar{\Phi}_{i j}^{l s}= \\
& {\left[\begin{array}{cccccc}
\bar{\Phi}_{11 i l} & \bar{\Phi}_{12 i l s} & \bar{Z}_{1 i} & 0 & \bar{\Phi}_{15 i l} & \bar{G}_{i}^{\mathrm{T}} \\
* & \bar{\Phi}_{22 i l s} & 0 & 0 & \bar{\Phi}_{25 i l s} & 0 \\
* & * & -\bar{Q}_{2 i}-\bar{Z}_{1 i} & 0 & 0 & 0 \\
* & * & * & -\bar{Q}_{3 i} & 0 & 0 \\
* & * & * & * & \bar{\Phi}_{55 i} & 0 \\
* & * & * & * & * & \bar{\Phi}_{66 i j}
\end{array}\right]} \\
& \bar{\Phi}_{11 i l}=\bar{Q}_{1 i}+\bar{Q}_{2 i}+\bar{Q}_{3 i}+\mu_{1} \operatorname{sym}\left(A_{i}^{l} \bar{G}_{i}\right)-\bar{Z}_{1 i}+\mu_{1}^{2} I \\
& \bar{\Phi}_{12 i l s}=\mu_{2} \bar{G}_{i}^{\mathrm{T}}\left(A_{i}^{l}\right)^{\mathrm{T}}+\mu_{1} B_{i}^{l} Y_{i}^{s} C_{2 i} \\
& \bar{\Phi}_{22 i l s}=\mu_{2} \operatorname{sym}\left(B_{i}^{l} Y_{i}^{s} C_{2 i}\right)-\left(1-h_{d i}\right) \bar{Q}_{1 i}+\mu_{2}^{2} I \\
& \bar{\Phi}_{15 i l}=\bar{P}_{i}-\mu_{1} \bar{G}_{i}+\mu_{3} \bar{G}_{i}^{\mathrm{T}}\left(A_{i}^{l}\right)^{\mathrm{T}} \\
& \bar{\Phi}_{25 i l s}=-\mu_{2} \bar{G}_{i}+\mu_{3} C_{2 i}^{\mathrm{T}}\left(Y_{i}^{s}\right)^{\mathrm{T}}\left(B_{i}^{l}\right)^{\mathrm{T}} \\
& \bar{\Phi}_{55 i}=\eta_{1 i}^{2} \bar{Z}_{1 i}-\mu_{3} \operatorname{sym}\left(\bar{G}_{i}\right)+\mu_{3}^{2} I \\
& \bar{\Phi}_{66 i j}=-\left(3 J \sum_{j=1}^{J} \hat{f}_{j i}^{2}\left\|\hat{B}_{j}\right\|^{2}\right)^{-1} I \\
& \bar{G}_{i}=V_{i}\left[\begin{array}{cc}
\hat{G}_{11 i} & 0 \\
\hat{G}_{21 i} & \hat{G}_{22 i}
\end{array}\right] V_{i}^{\mathrm{T}} .
\end{aligned}
$$

Then, the desired controller gains are given by $K_{i}^{s}=$ $Y_{i}^{s} W_{i} S_{i} \hat{G}_{11 i}^{-1} S_{i}^{-1} W_{i}^{\mathrm{T}}$, where $W_{i}, S_{i}$ and $V_{i}$ are derived from singular value decomposition (SVD) of $C_{2 i}$.

Proof. Under the conditions of the Theorem 1, a feasible solution satisfies the condition $\bar{\Phi}_{55 i}<0$, which implies that $\bar{G}_{i}$ is nonsingular. Define $G_{i}=\bar{G}_{i}^{-1}, \bar{P}_{i}=\bar{G}_{i}^{\mathrm{T}} P_{i} \bar{G}_{i}$, $\bar{Q}_{1 i}=\bar{G}_{i}^{\mathrm{T}} Q_{1 i} \bar{G}_{i}, \bar{Q}_{2 i}=\bar{G}_{i}^{\mathrm{T}} Q_{2 i} \bar{G}_{i}, \bar{Q}_{3 i}=\bar{G}_{i}^{\mathrm{T}} Q_{3 i} \bar{G}_{i}$ and $\bar{Z}_{1 i}=\bar{G}_{i}^{\mathrm{T}} Z_{1 i} \bar{G}_{i}$

Assume that $C_{2 i}$ is full rank, then the SVD decomposition exists such that $W_{i}^{\mathrm{T}} C_{2 i} V_{i}=\left[\begin{array}{ll}S_{i} & 0\end{array}\right]$ and $\bar{G}_{i}=$ $V_{i}\left[\begin{array}{cc}\hat{G}_{11 i} & 0 \\ \hat{G}_{21 i} & \hat{G}_{22 i}\end{array}\right] V_{i}^{\mathrm{T}}$. It is obtained that

$$
\begin{aligned}
C_{2 i} \bar{G}_{i}= & W_{i}\left[\begin{array}{ll}
S_{i} & 0
\end{array}\right] V_{i}^{\mathrm{T}} V_{i}\left[\begin{array}{cc}
\hat{G}_{11 i} & 0 \\
\hat{G}_{21 i} & \hat{G}_{22 i}
\end{array}\right] V_{i}^{\mathrm{T}}= \\
& W_{i}\left[\begin{array}{ll}
S_{i} \hat{G}_{11 i} & 0
\end{array}\right] V_{i}^{\mathrm{T}}= \\
& W_{i} S_{i} \hat{G}_{11 i} S_{i}^{-1} W_{i}^{\mathrm{T}} W_{i}\left[\begin{array}{ll}
S_{i} & 0
\end{array}\right] V_{i}^{\mathrm{T}}=\hat{G}_{i} C_{2 i} .
\end{aligned}
$$

By letting $Y_{i}^{l}=K_{i}^{l} \hat{G}=K_{i}^{l} W_{i} S_{i} \hat{G}_{11 i} S_{i}^{-1} W_{i}^{\mathrm{T}}$, using Schur complement and applying a congruence transformation to (25) and (26) by $\operatorname{diag}\left\{G_{i}, G_{i}, G_{i}, G_{i}, G_{i}\right\}$, we find that the 
condition (14) holds considering (4) and (11). Thus, there exists a fuzzy controller (7) such that the closed-loop system (13) is asymptotically stable.

\section{Simulation results}

Example 1. To show the effectiveness of the proposed approach, we consider the numerical example given in [4], which is composed of two subsystems $S_{1}$ and $S_{2}$ described respectively by

$$
\left\{\begin{array}{l}
\dot{x}_{1}(t)=\sum_{l=1}^{2} h_{1}^{l}\left(\theta_{1}(t)\right)\left[A_{1}^{l} x_{1}(t)+B_{1}^{l} u_{1}(t)+\sum_{j=1}^{J} f_{1 j}\left(x_{j}(t)\right)\right] \\
y_{1}(t)=C_{21} x_{1}(t)
\end{array}\right.
$$

with

$$
\begin{aligned}
& A_{1}^{1}=\left[\begin{array}{ccc}
-6 & 6 & 0 \\
0.5 & -3 & 1 \\
0 & 0.2 & -1
\end{array}\right], \quad A_{1}^{2}=\left[\begin{array}{ccc}
-1 & 0.1 & 0 \\
-0.2 & -2 & 0 \\
0.3 & 0 & -1
\end{array}\right] \\
& B_{1}^{1}=\left[\begin{array}{ll}
2 & 1 \\
1 & 1 \\
1 & 1
\end{array}\right], \quad B_{1}^{2}=\left[\begin{array}{ll}
1 & 2 \\
1 & 2 \\
1 & 1
\end{array}\right] \\
& C_{21}=\left[\begin{array}{lll}
1 & 0.1 & 0.1 \\
0.1 & 0.2 & 0.1
\end{array}\right], \quad f_{11}=0 \\
& f_{12}=\left[\begin{array}{ll}
0.02 & 0.01 \\
0.01 & 0.4 \\
0.01 & 0.1
\end{array}\right]\left\|x_{2}\right\| \\
& h_{1}^{1}\left(x_{1}(t)\right)=\sin ^{2}\left(x_{11}(t)\right), \quad h_{1}^{2}\left(x_{1}(t)\right)=\cos ^{2}\left(x_{11}(t)\right) .
\end{aligned}
$$

For subsystem $S_{2}$,

$$
\left\{\begin{array}{l}
\dot{x}_{2}(t)=\sum_{l=1}^{2} h_{2}^{l}\left(\theta_{2}(t)\right)\left[A_{2}^{l} x_{2}(t)+B_{2}^{l} u_{2}(t)+\sum_{j=1}^{J} f_{2 j}\left(x_{j}(t)\right)\right] \\
y_{2}(t)=C_{22} x_{2}(t)
\end{array}\right.
$$

with

$$
\begin{aligned}
& A_{2}^{1}=\left[\begin{array}{cc}
-1 & 0 \\
0 & -1
\end{array}\right], \quad A_{2}^{2}=\left[\begin{array}{cc}
-2 & 0 \\
0 & -1
\end{array}\right] \\
& B_{2}^{1}=\left[\begin{array}{l}
2 \\
1
\end{array}\right], \quad B_{2}^{2}=\left[\begin{array}{l}
2 \\
2
\end{array}\right] \\
& C_{22}=\left[\begin{array}{cc}
1 & 0 \\
0.1 & 1
\end{array}\right], \quad f_{21}=\left[\begin{array}{lll}
0.01 & 0.01 & 0.01 \\
0.02 & 0.01 & 0.1
\end{array}\right]\left\|x_{1}\right\| \\
& f_{22}=0, \quad h_{2}^{1}\left(x_{2}(t)\right)=\sin ^{2}\left(x_{21}(t)\right) \\
& h_{2}^{2}\left(x_{2}(t)\right)=\cos ^{2}\left(x_{21}(t)\right) .
\end{aligned}
$$

The network-related parameters for each subsystem $S_{i}$ are assumed as $T_{e}=3 \mathrm{~ms}$, the minimum delay $\eta_{1 i}=4 \mathrm{~ms}$, the maximum delay $\eta_{2 i}=20 \mathrm{~ms}$ and the maximum number of packet dropouts is $\bar{\sigma}_{i}=3$. The time varying delays between the sensors and controller as well as between controller and actuator are generated randomly such as $\min \left(\tau_{s c i}+\tau_{c a i}\right) \geq \eta_{1 i}$, and $\max \left(\tau_{s c i}+\tau_{c a i}+\left(\bar{\sigma}_{i}+1\right) T_{e}\right) \leq \eta_{2 i}$, and packet dropouts are also generated randomly such as $\max \left(N_{e}\right) \leq 3$, where $N_{e}$ is the number of packet dropouts, $h_{d i}=0.1, \mu_{1}=1, \mu_{2}=0.3$ and $\mu_{3}=0.5$.

By Theorem 1, we find a feasible solution as $K_{1}^{1}=$ $\left[\begin{array}{cc}1.2494 & -3.3911 \\ -1.3490 & 3.2305\end{array}\right], \quad K_{1}^{2}=\left[\begin{array}{cc}0.0596 & 0.5105 \\ -0.1445 & -0.5467\end{array}\right]$ for subsystem $S_{1}$, and $K_{2}^{1}=\left[\begin{array}{ll}-0.0559 & -0.0221\end{array}\right], K_{2}^{2}=$ $\left[\begin{array}{ll}-0.0311 & -0.0564\end{array}\right]$ for subsystem $S_{2}$.

For simulation, initial conditions are $x_{1}(0)=$ $\left[\begin{array}{lll}1 & 0.5 & -1\end{array}\right]^{\mathrm{T}}$ and $x_{2}(0)=\left[\begin{array}{ll}2 & -2\end{array}\right]^{\mathrm{T}}$.

The state variables evolution of NCSs and control inputs are shown in Figs. 2-4 from which, we can note that all states converge to zero. Figs. 5 and 6 show the delays introduced by the network and packet loss data which are randomly generated. Therefore, according to Theorem 1, the closed-loop overall fuzzy large-scale system composed of two subsystems $S_{1}$ and $S_{2}$ is asymptotically stable. The simulation results are consistent with the analysis and support the effectiveness of the developed design strategy.

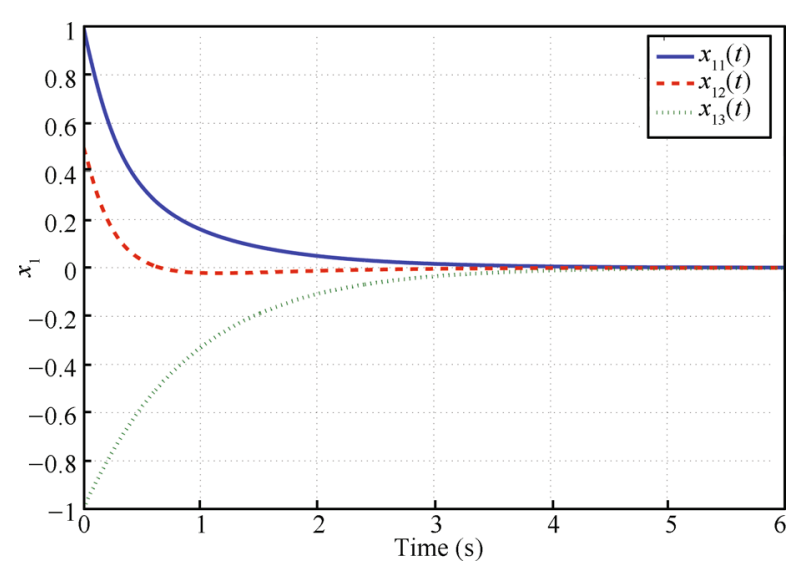

Fig. 2 Response of state $x$ in the $S_{1}$

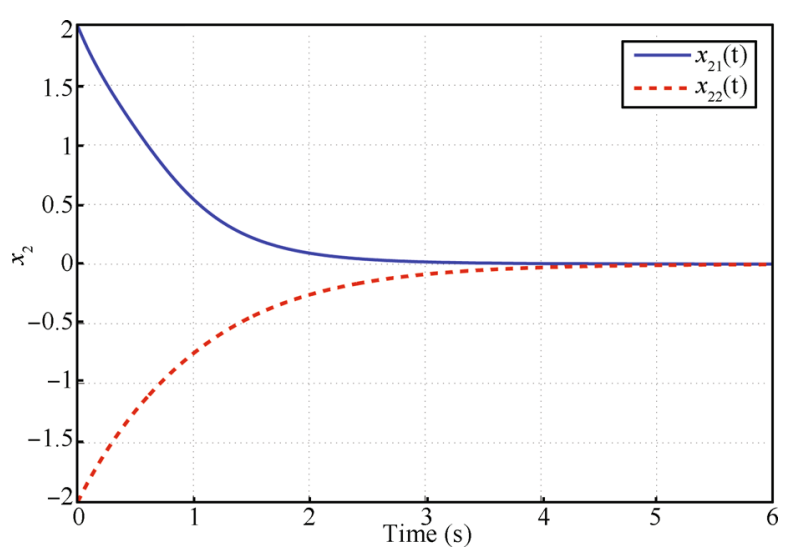

Fig. 3 Response of state $x$ in the $S_{2}$ 


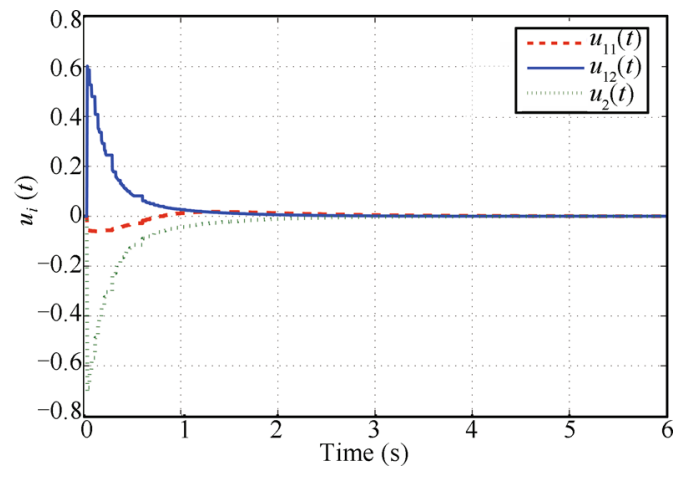

Fig. 4 Evolution of control input signals $u_{i}(t)$

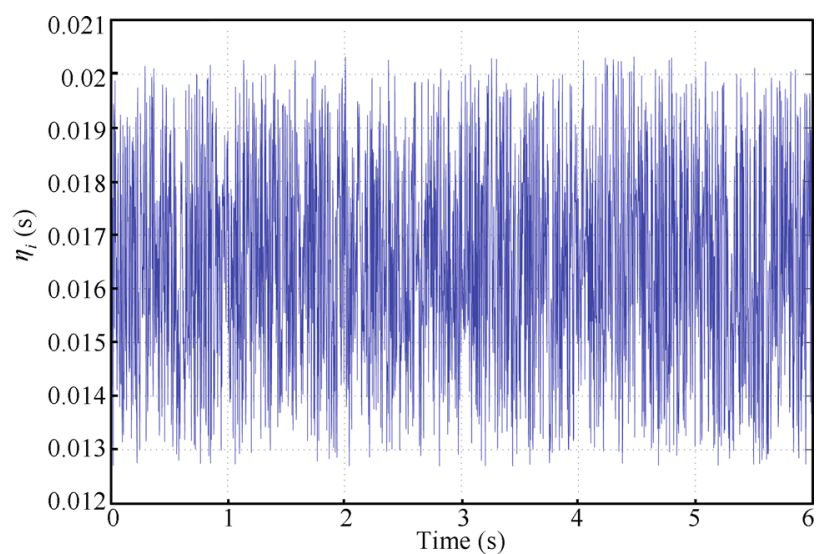

Fig. 5 Delay induced by communication networks

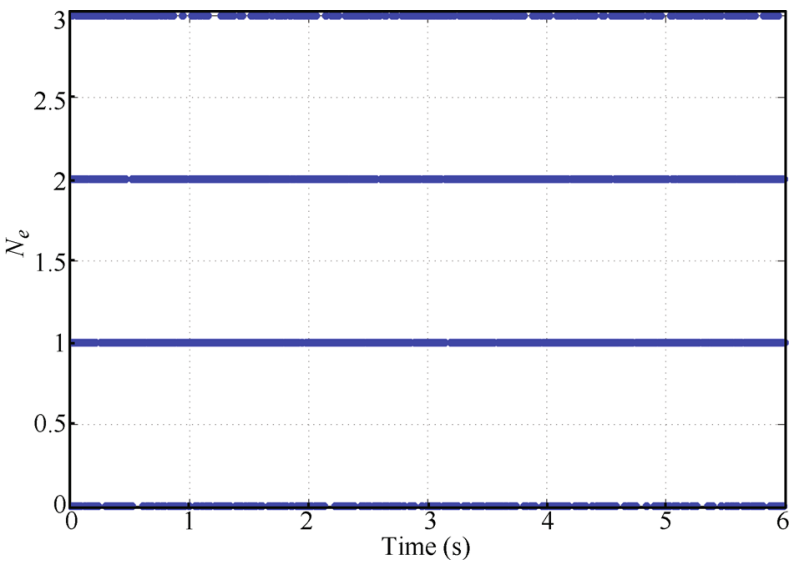

Fig. 6 Data packets dropout

Example 2. We consider the same large-scale system $S$ composed of three fuzzy subsystems $S_{i}, i=1,2,3$, as that in $[20]$.

For subsystem $S_{1}$ :

Rule 1 :

$$
\begin{aligned}
& \text { If } x_{11}(t) \text { is small and } x_{12}(t) \text { is big } \\
& \text { then } \dot{x}_{1}(t)=A_{1}^{1} x_{1}(t)+B_{1}^{1} u_{1}+\sum_{j=1}^{3} f_{1 j}\left(x_{j}(t)\right) \\
& \qquad y_{1}(t)=C_{11} x_{1}(t) .
\end{aligned}
$$

Rule 2:

$$
\begin{aligned}
& \text { If } x_{11}(t) \text { is small and } x_{12}(t) \text { is small } \\
& \text { then } \dot{x}_{1}(t)=A_{1}^{2} x_{1}(t)+B_{1}^{2} u_{1}+\sum_{j=1}^{3} f_{1 j}\left(x_{j}(t)\right) \\
& \qquad y_{1}(t)=C_{11} x_{1}(t) .
\end{aligned}
$$

Rule 3 :

If $x_{11}(t)$ is big and $x_{12}(t)$ is small

$$
\text { then } \begin{aligned}
\dot{x}_{1}(t) & =A_{1}^{3} x_{1}(t)+B_{1}^{3} u_{1}+\sum_{j=1}^{3} f_{1 j}\left(x_{j}(t)\right) \\
y_{1}(t) & =C_{11} x_{1}(t) .
\end{aligned}
$$

For these rules,

$$
\begin{aligned}
A_{1}^{1} & =\left[\begin{array}{cc}
-2 & 3 \\
1.5 & -2.2
\end{array}\right], \quad A_{1}^{2}=\left[\begin{array}{cc}
-4 & 3 \\
3 & -2
\end{array}\right] \\
A_{1}^{3} & =\left[\begin{array}{cc}
-2 & 3 \\
-6 & -11
\end{array}\right], \quad B_{1}^{1}=\left[\begin{array}{c}
0.15 \\
0.1
\end{array}\right] \\
B_{1}^{2} & =\left[\begin{array}{l}
0.6 \\
0.4
\end{array}\right], \quad B_{1}^{3}=\left[\begin{array}{l}
0.3 \\
0.2
\end{array}\right] \\
C_{11} & =\left[\begin{array}{ll}
1 & 0 \\
0.1 & 1
\end{array}\right], \quad f_{11}=0 \\
f_{12} & =\left[\begin{array}{l}
0.08 \\
0.05
\end{array}\right]\left\|x_{2}\right\|, \quad f_{13}=\left[\begin{array}{l}
0.09 \\
0.06
\end{array}\right]\left\|x_{3}\right\| .
\end{aligned}
$$

For subsystem $S_{2}$ :

Rule 1 :

If $x_{21}(t)$ is small and $x_{22}(t)$ is small

$$
\text { then } \begin{aligned}
\dot{x}_{2}(t) & =A_{2}^{1} x_{2}(t)+B_{2}^{1} u_{2}+\sum_{j=1}^{3} f_{2 j}\left(x_{j}(t)\right) \\
y_{2}(t) & =C_{11} x_{2}(t) .
\end{aligned}
$$

Rule 2 :

$$
\begin{aligned}
& \text { If } x_{21}(t) \text { is big and } x_{22}(t) \text { is small } \\
& \text { then } \dot{x}_{2}(t)=A_{2}^{2} x_{2}(t)+B_{2}^{2} u_{2}+\sum_{j=1}^{3} f_{2 j}\left(x_{j}(t)\right) \\
& y_{2}(t)=C_{11} x_{2}(t) .
\end{aligned}
$$

For these rules,

$$
\begin{aligned}
& A_{2}^{1}=\left[\begin{array}{cc}
-3 & 1 \\
5 & -3
\end{array}\right], A_{2}^{2}=\left[\begin{array}{cc}
-2 & 1 \\
3 & -0.3
\end{array}\right] \\
& B_{2}^{1}=\left[\begin{array}{l}
0.1 \\
0.6
\end{array}\right], B_{2}^{2}=\left[\begin{array}{l}
0.2 \\
1.2
\end{array}\right] \\
& f_{21}=\left[\begin{array}{l}
0.02 \\
0.12
\end{array}\right]\left\|x_{1}\right\|, f_{22}=0 \\
& f_{23}=\left[\begin{array}{l}
0.06 \\
0.36
\end{array}\right]\left\|x_{3}\right\| .
\end{aligned}
$$

For subsystem $S_{3}$ : 
Rule 1 :

If $x_{31}(t)$ is big and $x_{32}(t)$ is big

$$
\text { then } \begin{aligned}
\dot{x}_{3}(t) & =A_{3}^{1} x_{3}(t)+B_{3}^{1} u_{3}+\sum_{j=1}^{3} f_{3 j}\left(x_{j}(t)\right) \\
y_{3}(t) & =C_{11} x_{3}(t) .
\end{aligned}
$$

Rule 2 :

If $x_{31}(t)$ is small and $x_{32}(t)$ is big

$$
\text { then } \begin{aligned}
\dot{x}_{3}(t) & =A_{3}^{2} x_{3}(t)+B_{3}^{2} u_{3}+\sum_{j=1}^{3} f_{3 j}\left(x_{j}(t)\right) \\
y_{3}(t) & =C_{11} x_{3}(t) .
\end{aligned}
$$

For these rules,

$$
\begin{aligned}
A_{3}^{1} & =\left[\begin{array}{cc}
-3 & 1 \\
4 & -2
\end{array}\right], A_{3}^{2}=\left[\begin{array}{cc}
-2 & 1 \\
3 & -1
\end{array}\right] \\
B_{3}^{1} & =\left[\begin{array}{l}
0.6 \\
0.8
\end{array}\right], B_{3}^{2}=\left[\begin{array}{l}
0.3 \\
0.4
\end{array}\right] \\
f_{31} & =\left[\begin{array}{l}
0.48 \\
0.64
\end{array}\right]\left\|x_{1}\right\|, \quad f_{32}=\left[\begin{array}{l}
0.24 \\
0.32
\end{array}\right]\left\|x_{2}\right\| \\
f_{33} & =0 .
\end{aligned}
$$

It is seen that all $f_{i j}$ satisfy the matching condition (2) with $\hat{f}_{11}^{1}=\hat{f}_{11}^{2}=\hat{f}_{11}^{3}=0, \hat{f}_{12}^{1}=0.5, \hat{f}_{12}^{2}=0.125, \hat{f}_{12}^{3}=$ $0.25, \hat{f}_{13}^{1}=0.6, \hat{f}_{13}^{2}=0.15$ and $\hat{f}_{13}^{3}=0.3$ for subsystem $S_{1}$. All $f_{i j}$ satisfy (2) with $\hat{f}_{21}^{1}=0.2, \hat{f}_{21}^{2}=0.1, \hat{f}_{22}^{1}=\hat{f}_{22}^{2}=$ $0, \hat{f}_{23}^{1}=0.6$ and $\hat{f}_{23}^{2}=0.3$ for subsystem $S_{2}$, All $f_{i j}$ satisfy (2) with $\hat{f}_{31}^{1}=0.8, \hat{f}_{31}^{2}=1.6, \hat{f}_{32}^{1}=0.4, \hat{f}_{32}^{2}=0.8$ and $\hat{f}_{33}^{1}=\hat{f}_{33}^{2}=0$ for subsystem $S_{3}$.

The membership functions of each state are shown in Fig. 1 of [20].

The network-related parameters for each subsystem $S_{i}$ are assumed as $T_{e}=5 \mathrm{~ms}$, the minimum delay $\eta_{1 i}=6 \mathrm{~ms}$, the maximum delay $\eta_{2 i}=20 \mathrm{~ms}$ and the maximum number of packet dropouts is $\bar{\sigma}_{i}=2$. The time varying delays between the sensors and controller as well as between controller and actuator are generated randomly such as $\min \left(\tau_{s c i}+\tau_{c a i}\right) \geq \eta_{1 i}$, and $\max \left(\tau_{s c i}+\tau_{c a i}+\left(\bar{\sigma}_{i}+1\right) T_{e}\right) \leq \eta_{2 i}$ and packet dropouts are also generated randomly such as $\max \left(N_{e}\right) \leq 2, h_{d i}=0.1, \mu_{1}=1, \mu_{2}=0.5$ and $\mu_{3}=0.9$.

Applying Theorem 1, the solutions of LMIs can be obtained as $K_{1}^{1}=\left[\begin{array}{ll}-4.2341 & -4.6081\end{array}\right], \quad K_{1}^{2}=$ $\left[\begin{array}{ll}-1.1609 & -1.0426\end{array}\right]$ and $K_{1}^{3}=\left[\begin{array}{ll}0.6774 & 0.2271\end{array}\right]$ for subsystem $S_{1}, K_{2}^{1}=\left[\begin{array}{ll}-2.2344 & -2.9514\end{array}\right]$ and $K_{2}^{2}=$ $\left[\begin{array}{ll}-1.8578 & -1.5973\end{array}\right]$ for subsystem $S_{2}$, and $K_{3}^{1}=$ $\left[\begin{array}{ll}-0.8119 & -0.8278\end{array}\right]$ and $K_{3}^{2}=\left[\begin{array}{ll}-1.9309 & -1.6042\end{array}\right]$ for
subsystem $S_{3}$.

For simulation, initial conditions are $x_{1}(0)=$ $\left.\begin{array}{ll}1.5 & -1\end{array}\right]^{\mathrm{T}}, \quad x_{2}(0)=\left[\begin{array}{ll}-0.5 & 0.5\end{array}\right]^{\mathrm{T}}$ and $x_{3}(0)=$
The state variable evolution of NCSs and control inputs are shown in Figs. 7-10 from which, we can note that all states converge to zero. Therefore, according to Theorem 1 , the closed-loop overall fuzzy large-scale system composed of three subsystems $S_{1}, S_{2}$ and $S_{3}$ is asymptotically stable. Thus, we have shown that the proposed decentralized static output feedback controller makes the nonlinear interconnected system in network communication exhibit asymptotic stability.

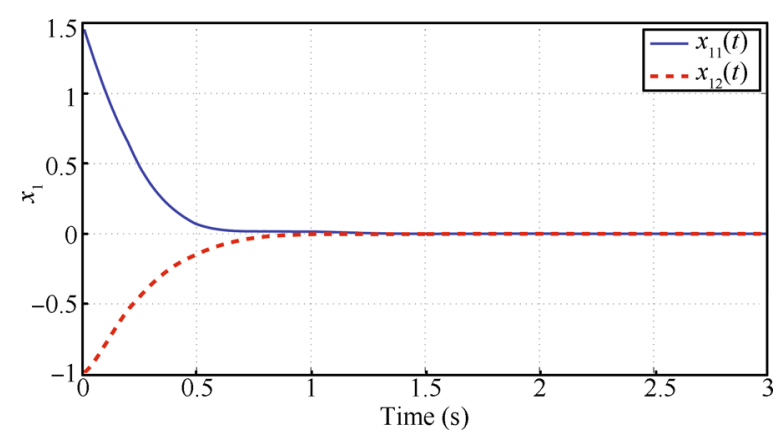

Fig. 7 State responses $x$ in $S_{1}$

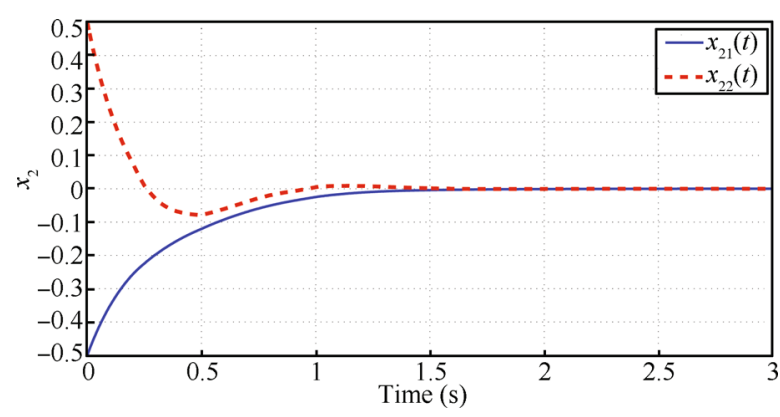

Fig. 8 State responses $x$ in $S_{2}$

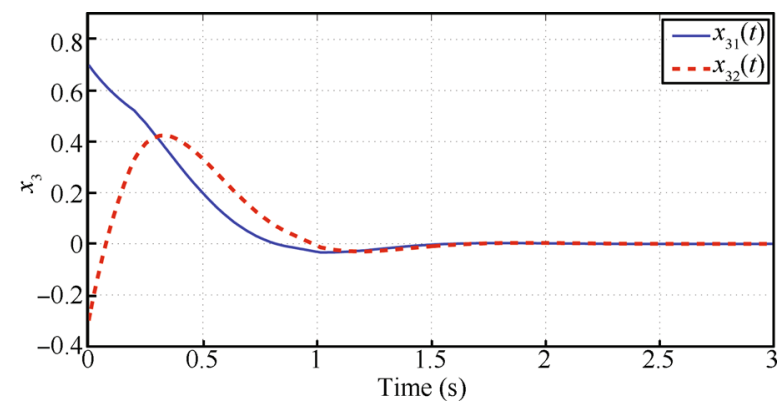

Fig. 9 State responses $x$ in $S_{3}$

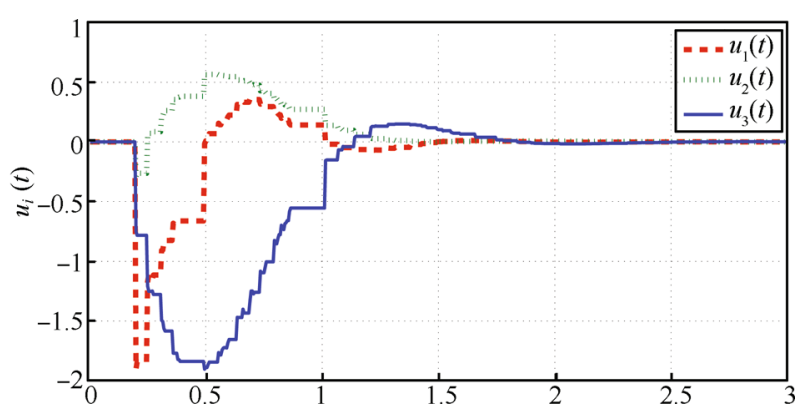

Fig. 10 Control signal trajectories $u_{i}(t)$ 


\section{Conclusions}

In this paper, based on Lyapunov-Krasovskii functional, new stabilization conditions have been established for networked controlled large-scale system. Furthermore, using these conditions in presence of the delay and data packets dropout in the network communication, networked fuzzy static output feedback controller gains have been obtained. The simulation results are shown to prove the advantages of the developed method.

\section{References}

[1] M. Cocetti, L. Sabattini, C. Secchi, C. Fantuzzi. Decentralized control strategy for the implementation of cooperative dynamic behaviors in networked systems. In Proceedings of IEEE/RSJ International Conference on Intelligent Robots and Systems, IEEE, Tokyo, Japan, pp. 5902-5907, 2013.

[2] Y. Hu, N. H. El-Farra. Quasi-decentralized output feedback model predictive control of networked process systems with forecast-triggered communication. In Proceedings of American Control Conference, IEEE, Washington, USA, pp. 2612-2617, 2013.

[3] Z. Liu, X. Chen, Z. Y. Jiang, L. F. Qiao, X. H. Guan. Asynchronous latency analysis on decentralized iterative algorithms for large scale networked systems. In Proceedings of the 32nd Chinese Control Conference, IEEE, Xi'an, China, pp. 6900-6905, 2013.

[4] K. Guelton, N. Manamanni, D. Jabri. H-infinity decentralized Static Output Feedback controller design for large scale Takagi-Sugeno systems. In Proceedings IEEE International Conference on Fuzzy Systems, IEEE, Barcelona, Spain, pp. 1-7, 2010.

[5] C. C. Hua, S. X. Ding. Decentralized networked control system design using T-S fuzzy approach. IEEE Transactions on Fuzzy Systems, vol. 20, no. 1, pp. 9-21, 2012.

[6] J. H. Park. Robust nonfragile decentralized controller design for uncertain large-scale interconnected systems with time-delays. Journal of Dynamic Systems, Measurement, and Control, vol. 124, no. 2, pp. 332-336, 2002.

[7] J. H. Park. Design of robust decentralized dynamic controller for uncertain large-scale interconnected systems with time-delays. IEICE Transactions on Fundamentals of Electronics, Communications and Computer Sciences, vol. E84A, no. 7, pp. 1747-1754, 2001.

[8] G. Scorletti, G. Duc. An LMI approach to dencentralized $H_{\infty}$ control. International Journal of Control, vol. 74, no. 3, pp. 211-224, 2001.

[9] X. S. Xiao, Z. Z. Mao. Decentralized guaranteed cost stabilization of time-delay large-scale systems based on reducedorder observers. Journal of the Franklin Institute, vol. 348, no. 9, pp. 2689-2700, 2011.
[10] C. Latrach, M. Kchaou, A. El Hajjaji, A. Rabhi. $H_{\infty}$ fuzzy networked control for vehicle lateral dynamics. In Proceedings of the 21st Mediterranean Conference on Control \& Automation, IEEE, Chania, Greece pp. 25-28, 2013.

[11] C. Latrach, M. Kchaou, A. El Hajjaji, A. Rabhi. Robust $H_{\infty}$ fuzzy networked control for vehicle lateral dynamics. In Proceedings of the 16th International IEEE Annual Conference on Intelligent Transportation Systems, IEEE, The Hague, Holland, pp. 6-9, 2013.

[12] C. Peng, Y. C. Tian, M. O. Tadé. State feedback controller design of networked control systems with interval time-varying delay and nonlinearity. International Journal of Robust and Nonlinear Control, vol. 18, no. 12, pp. 12851301, 2008.

[13] Y. Shi, H. Fang, M. Yan. Kalman filter-based adaptive control for networked systems with unknown parameters and randomly missing outputs. International Journal of Robust and Nonlinear Control, vol. 19, no. 18, pp. 1976-1992, 2009.

[14] J. Wu, L. Q. Zhang, T. W. Chen. Model predictive control for networked control systems. International Journal of Robust and Nonlinear Control, vol. 19, no. 9, pp.1016-1035, 2009 .

[15] L. G. Wu, J. Lam, X. M. Yao, J. L. Xiong. Robust guaranteed cost control of discrete-time networked control systems. Optimal Control Applications and Methods, vol. 32, no. 1, pp. 95-112, 2011

[16] H. J. Yang, Y. Q. Xia, P. Shi. Stabilization of networked control systems with nonuniform random sampling periods. International Journal of Robust and Nonlinear Control, vol. 21, no. 5, pp. 501-526, 2011.

[17] B. Yu, Y. Shi, Y. Lin. Discrete-time $H_{2}$ output tracking control of wireless networked control systems with Markov communication models. Wireless Communications and Mobile Computing, vol.11, no. 8, pp.1107-1116, 2011.

[18] M. Kchaou, A. Toumi. Fuzzy network-based control for a class of TS fuzzy systems with limited communication. Transactions on Systems, Signals and Devices, vol. 9, pp. 118, 2014.

[19] H. Zhang, Y. Shi, A. S. Mehr. Robust weighted $H_{\infty}$ filtering for networked systems with intermittent measurements of multiple sensors. International Journal of Adaptive Control and Signal Processing, vol. 25, no. 4, pp. 313-330, 2011.

[20] W. J. Wang, W. W. Lin. Decentralized PDC for large-scale T-S fuzzy systems. IEEE Transactions on Fuzzy Systems, vol. 13 , no. 6 , pp. $779-786,2005$.

[21] K. Zhou, P. Khargonedkar. Robust stabilization of linear systems with norm-bounded time-varying uncertainty. Systems \& Control Letters, vol. 10, no. 1, pp. 17-20, 1988. 
[22] J. M. Gomes da Silva Jr, A. Seuret, E. Fridman, J. P. Richard. Stabilisation of neutral systems with saturating control inputs. International Journal of Systems Science, vol. 42, no. 7, pp. 1093-1103, 2011.

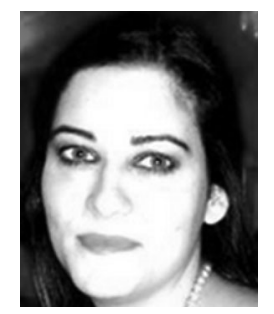

Chedia Latrach received the M. Sc. degree in electrical engineering from the $\mathrm{Na}$ tional Engineering School of Sfax, Tunisia in 2011. She is currently a Ph. D. candidate in the University of Picardie Jules Verne, France.

Her research interests include fuzzy control, vehicle dynamics, networked control and decentralized control.

E-mail: chedia.latrach@yahoo.fr (Corresponding author) ORCIDiD: 0000-0002-0201-9819

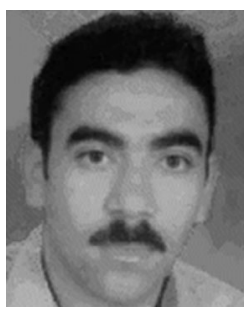

Mourad Kchaou received the Ph. D. degree in automatic and industrial computing from the National School of Engineering, University of Sfax, Tunisia in 2009. He is now an assistant professor in the High Institute of Applied Sciences and Technology University of Sousse, Tunisia. He is a member of the Laboratory of Sciences and Techniques of Automation Control and Computer Engineering, National Engineering School of Sfax, Tunisia.

His research interests include fuzzy control, time-delay systems, descriptor systems, with particular attention paid to nonlinear systems represented by multiple-models.

E-mail: mouradkchaou@gmail.com

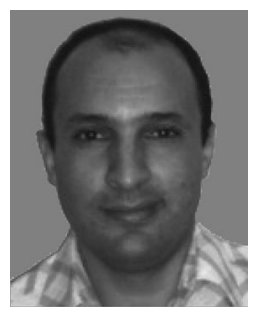

Abdelhamid Rabhi received the $\mathrm{Ph}$. D. degree in observation and control of nonlinear and complex systems in 2005. Since 2006, he has been an associate professor at the Faculty of Sciences, University of Picardie Jules Verne, France, and a researcher in the Modeling, Information and Systems (MIS) Laboratory, Amiens, France.

His research interests include fuzzy control, vehicle dynamics and renewable energy systems.

E-mail: abdelhamid.rabhi@u-picardie.fr

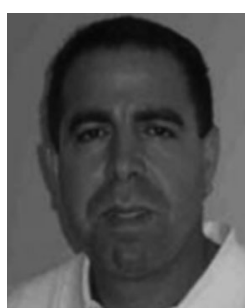

Ahmed El Hajjaji received the Ph. D. degree in automatic control from University of Picardie Jules Verne, France in 1993 and 2000 , respectively. He was an associate professor in the same university from 1994 to 2003. He is currently a full professor and director of the Electrical Engineering Department, University of Picardie Jules Verne, France. Since 2001, he has been the head of the research team of Control and Vehicle in Modeling, Information and Systems Laboratory, University of Picardie Jules Verne, France.

His research interests include fuzzy control, vehicle dynamics, fault tolerant control (FTC), neural networks, maglev systems, and renewable energy systems.

E-mail: ahmed.hajjaji@u-picardie.fr 